\title{
Advanced Image Enhancement Based on Wavelet \& Histogram Equalization for Medical Images
}

\author{
Yashu Rajput ${ }^{1}$, Vishwashvar Singh Rajput ${ }^{2}$, Anita Thakur ${ }^{3}$, Garima Vyas ${ }^{4}$ \\ 1,3,4, (Department of ECE, ASET, Amity University, Noida, India) \\ ${ }^{2}$ (Department of CSE, BIET, Jhansi, India)
}

\begin{abstract}
Image enhancement is used to produce high quality pictures like Medical images. As the quality of the image is very much depends on environmental effects like light, weather or equipment that we used to capture the picture, images may loose important information which is required to enhance the quality of an image. So we have many techniques to recover the lost data to improve the quality of the picture. In this paper, we are using non linear enhancement technique to increase the contrast level of an image like wavelet transform and Histogram Equalization. In this experiment we found that this method enhance the contrast level of an image.
\end{abstract}

Keywords: Image enhancement, Histogram equalization, Discrete wavelet transform (DWT),PSNR,MSE

\section{INTRODUCTION}

Image enhancement technology plays a vital role in medical imaging. The main purpose of image enhancement in medical imaging is used to provide better results, as Doctors always prefer high quality picture to diagnose the results .Image enhancement techniques are based on two broad categories, those are: Spatial domain methods, which operate directly on pixels, and Frequency domain methods, which operate on the Fourier transform of an image. So we can say that these techniques basically used to diagnose, display and amplify the image features. Here Frequency domain method mainly concentrating on the wavelet transform, the spatial domain method is concentrating on the histogram equalization method in image enhancement field.

Histogram equalization [1] method generally used to increase the contrast of many images, especially when the important data of that image is represented by close contrast values. Through this adjustment, the intensity can be better distributed across the histogram. Histogram equalization effectively spreading out the most frequent intensity values . This method is useful for both bright $\&$ dark regions in medical imaging. This method is simple, fast and we get acceptable results for many applications, these are the main advantages of this method. Disadvantage of this method is, it may increase the contrast of background noise, while it decreases the usable signal.

On the other hand, Wavelet transform technique is based on 2D-Discrete wavelet transform (2DDWT). A discrete wavelet transform (DWT) is another wavelet transform for which the wavelets are discretely sampled for numerical analysis and functional analysis. The advantage of DWT is, it captures both frequency and time information. Discrete wavelet transform (DWT) decompose signals into sub-bands with smaller bandwidths and slower sample rates namely Low-Low (LL), Low-High (LH), High-Low (HL), and High-High (HH). With this we get four sub-bands from one level of transform - first low-pass sub-band having the coarse approximation of the source image called LL sub-band, and three high pass sub-bands that exploit image details across different directions - HL for horizontal, $\mathrm{LH}$ for vertical and $\mathrm{HH}$ for diagonal details. After decompose these bands we can obtain high frequency components using these sub bands .So it will help to enhance the medical images. If we use both spatial field \& frequency domain field in the wavelet transform then we can obtain better enhanced image, as well as we can also reduce the noise in these high frequency sub-bands.

\subsection{HISTOGRAM EQUALIZATION}

\section{HISTOGRAM EQUALIZATION \& WAVELET TRANSFORM}

Histogram equalization techniques which modify the dynamic range \& contrast level of an image, such that its intensity histogram has a desired shape. Histogram equalization [2] operators can be employed non linear $\&$ non-monotonic transfer function for mapping between input and output images pixel intensity values . Histogram equalization employs a monotonic, non-linear mapping, which re-assigns the flat histogram (uniform distribution of intensities) for the different intensity value pixels in the output image as well as input image. This technique is using for image comparison \& correction in the non linear process. (Because it is using to enhance the image quality), which introduced by a digitizer or display system improvements. 


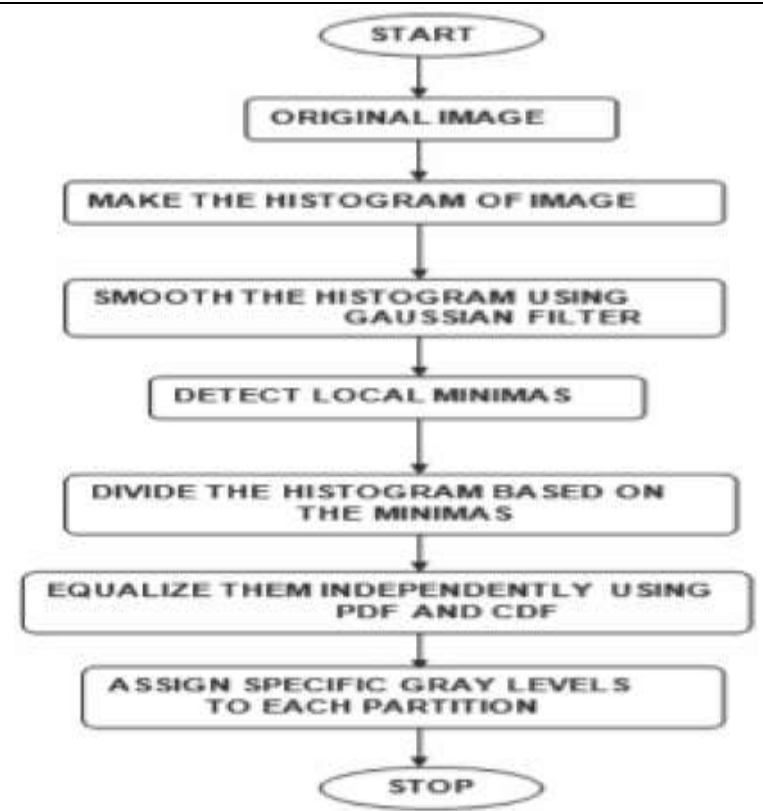

Figure .1 Basic steps to calculate the histogram of an image

If we transform the input image to get $s=T(r)$

Here $p_{s}$ and $p_{y}$ represents the probability density function of $\mathrm{s}$ and $\mathrm{r}$ respectively then $p_{s}$ can be represented by a simple formula

$$
p_{s}(s)=p_{r}(r) \frac{d r}{d s}
$$

where $r=T^{l}(s)$.

Consider the transformation

$$
s=T(r)=\int_{0}^{r} p_{r(w)} d w
$$

Given a transformation function $\mathrm{T}(\mathrm{r})$ we can get $p_{s}$ so that $p_{s}(s)$ follows almost uniform distribution which results in histogram equalized image.

Histogram equalization increases the intensity values up to its dynamic range as well as it flatten the histogram. In many images we found the satisfactory result using histogram equalization technique but sometimes it over enhance the image due to its global treatment

\subsection{WAVELET TRANSFORM}

Wavelet transform [3][4] is capable of providing the time and frequency information simultaneously. The frequency and time information of a signal .But sometimes we cannot know what spectral component exists at any given time instant. The best we can do is to investigate that what spectral components exist at any given interval of time.

Higher frequencies are better resolved in time, and lower frequencies are better resolved in frequency. This means that, a certain high frequency component can be located better in time (with less relative error) than a low frequency component $\&$ low frequency component can be located better in frequency compared to high frequency component.

\subsubsection{THE DISCRETE WAVELET TRANSFORM (DWT)}

The discrete wavelet transform (DWT) [5]is an implementation of the wavelet transform using a discrete set of the wavelet scales for numerical analysis and functional analysis. A time-scale representation of a digital signal is obtained using digital filtering techniques. In the discrete wavelet transform, filters of different cut-off frequencies are used to analyze the signal at different scales. If the wavelets are discretely sampled, the resultant coefficients are called as discrete wavelet transform (DWT)[6].

$$
f[n]=\frac{1}{\sqrt{M}} \sum_{k} W_{\varphi}\left[j_{0}, k\right] \varphi j_{0, k}[n]+\frac{1}{\sqrt{M}} \sum_{j=j_{0}}^{\infty} \sum_{k} W_{\Psi}[j, k] \Psi_{j, k}[n]
$$

We can simply take the inner product to obtain the wavelet coefficients 


$$
\begin{aligned}
& W_{\varphi}\left[j_{0}, k\right]=\frac{1}{\sqrt{M}} \sum_{n} f[n] \varphi_{j_{0 . k}}[n] \\
& W_{\Psi}[j, k]=\frac{1}{\sqrt{M}} \sum_{n} f[n] \Psi_{j_{k} k}[n] \quad \mathrm{j} \geq j_{0}
\end{aligned}
$$

The signal is computed by a series of high pass filters \& a series of low pass filters to analyze the high frequencies $\&$ the low frequencies of the discrete time domain signal, which is shown in figure-2. This is called the Mallat algorithm or Mallat-tree decomposition[7].

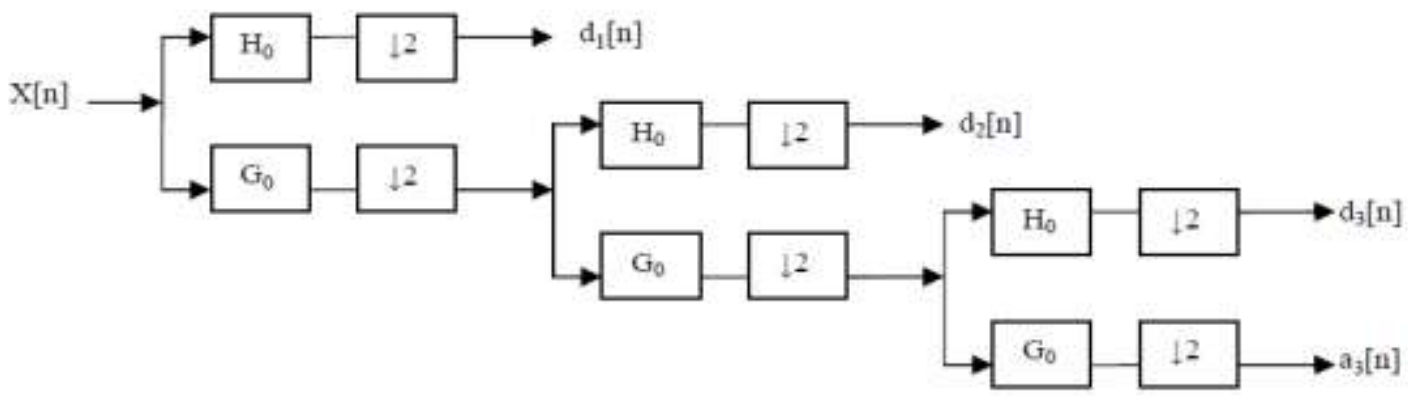

Figure.2 Three- level wavelet decomposition tree

In the figure.2, the signal is denoted by the sequence $\mathrm{x}[\mathrm{n}]$, where $\mathrm{n}$ is an integer. The low pass filter is denoted by G0 while the high pass filter is denoted by H0. At each level, the high pass filter produces detail information, $d[n]$, while the low pass filter associated with scaling function produces coarse approximations, $\mathrm{a}[\mathrm{n}]$.

This decomposition is repeated to further increase the frequency resolution and the approximation coefficients decomposed with high and low pass filters and then down-sampled. This is represented as a binary decomposition tree with nodes representing a sub-space with different time-frequency localization. The tree is known as a filter bank .

\subsection{MSE}

\section{III . Measurement Parameters (Mse \& Psnr)}

The average squared difference between the reference signal and distorted signal is called as the Mean squared error. It can be easily calculated by adding up the squared difference of pixel by pixel and dividing by the total pixel count [8].

Let $\mathrm{mxn}$ is a noise free monochrome image $\mathrm{I}$, and $\mathrm{K}$ is defined as the noisy approximation .Then the mean square error between these two signals is defined as:

$$
\mathrm{MSE}=\frac{1}{m \times n} \sum_{i=0}^{m-1} \sum_{j=0}^{n-1}\left[I\left(i_{j} j\right)-K\left(i_{j} j\right)\right]^{2}
$$

\subsection{PSNR}

The ratio between the reference signal and the distortion signal of an image is called as the peak signal to noise ratio, given in decibels. In general, a higher PSNR value should correlate to a higher quality image.

So PSNR is defined as:

$$
\text { PSNR }=10 \log _{10} \frac{L^{2}}{\text { MSE }}
$$

Here $\mathrm{L}$ reflects the the maximum possible pixel value of the image .If the $\mathrm{K}$ channel is encoded with a depth of 8 -bit, then $L=2^{\wedge} 8-1=255$. PSNR is usually expressed in terms of the decibels scale .If a signal to noise ratio is high then the mean square error will be minimum. 
IV. Results And Discussions

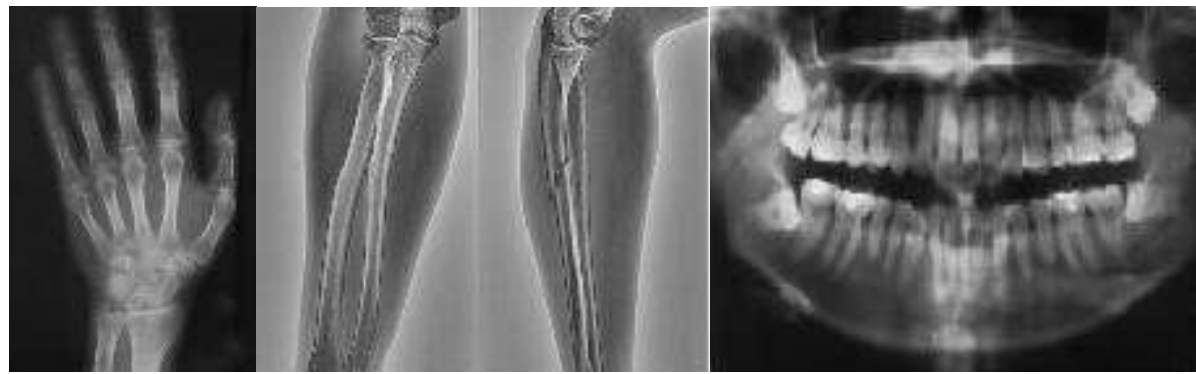

Figure.3 original image
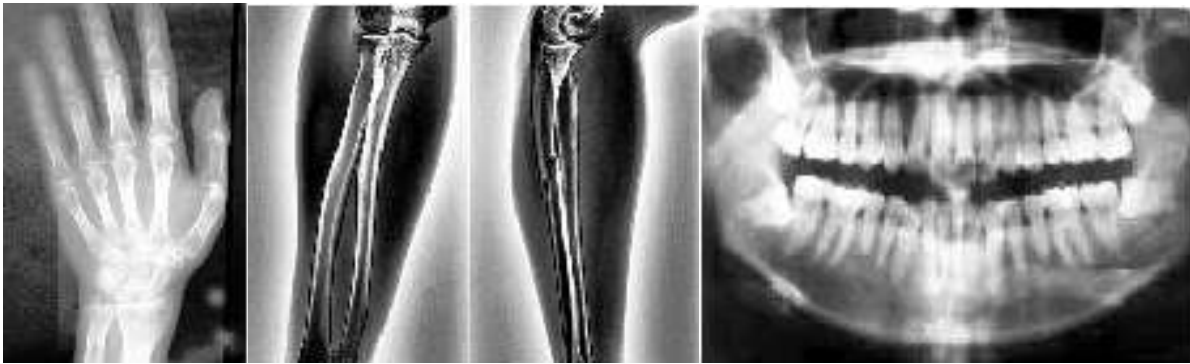

Figure.4 Enhanced image with histogram equalization
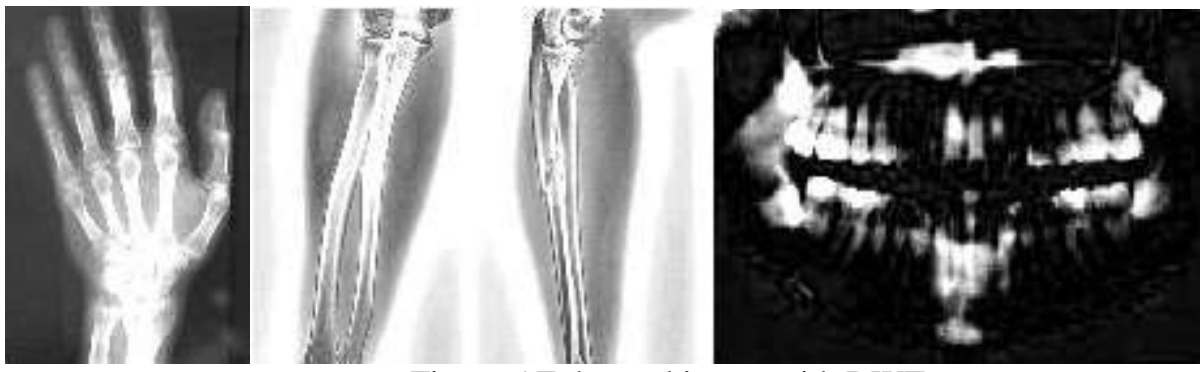

Figure.5 Enhanced image with DWT
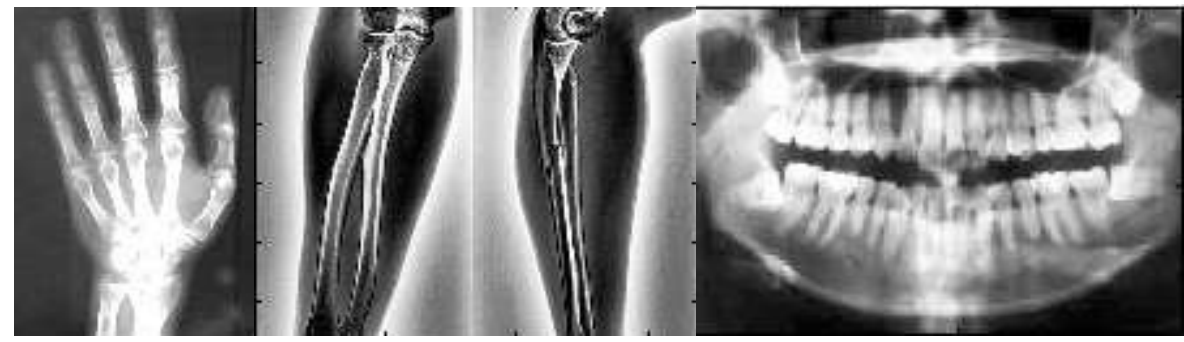

Figure.6 Enhanced image with preprocessed histogram equalization

\begin{tabular}{|c|c|c|c|}
\hline $\begin{array}{c}\text { Image } \\
\text { (BMP images) }\end{array}$ & Technique name & $\begin{array}{c}\text { MSE(For Recovered } \\
\text { Enhancement) }\end{array}$ & $\begin{array}{c}\text { PSNR(dB)(For Recovered } \\
\text { Enhancement) }\end{array}$ \\
\hline \multirow{2}{*}{$\begin{array}{c}\text { Hand. Jpg } \\
\mathbf{( 4 6 9 x 7 0 0 )}\end{array}$} & Histogram equalization & $5.4342 \mathrm{e}+003$ & 9.6665 \\
\cline { 2 - 4 } & DWT & 3.2720 & 41.8697 \\
\cline { 2 - 4 } & preprocessed histogram & $8.3631 \mathrm{e}-005$ & 87.7942 \\
\hline \multirow{2}{*}{$\begin{array}{c}\text { Legs. bmp } \\
\mathbf{( 3 3 8 x 2 7 8 )}\end{array}$} & Histogram equalization & $1.9219 \mathrm{e}+003$ & 14.1806 \\
\cline { 2 - 4 } & preprocessed histogram & 38.2930 & 90.1866 \\
\hline \multirow{2}{*}{$\begin{array}{c}\text { Teeth. Bmp } \\
(\mathbf{2 7 5 x 1 8 3 )}\end{array}$} & Histogram equalization & $3.2144 \mathrm{e}+005$ & 11.9469 \\
\cline { 2 - 4 } & DWT & 18.2275 & 34.4106 \\
\cline { 2 - 4 } & preprocessed histogram & $2.6123 \mathrm{e}-005$ & 92.8476 \\
\hline
\end{tabular}

TABLE .1 Comparisons based on MSE \& PSNR 
Proposed method is using for enhance the contrast level of the medical images. In this we are using the Simple non linear histogram equalization, Discrete wavelet transform \& the pre-processed histogram equalization technique, so we got better results using pre-processed histogram equalization technique, These results are compared with the help of MSE \& PSNR values.

Figure. 3 shows the original images of Hand, Legs \& Teeth. Figure. 4 shows the enhanced results using the histogram equalization technique, Figure.5 shows the results of discrete wavelet transform techniques \& Figure.6 shows the results of pre-processed histogram equalization technique.

So according to table we can say that the results of PSNR are increasing in the case of pre-processed histogram equalization technique and MSE is decreasing .So the images are much clear than the original images, .As per the figure.5, we can say that the wavelet method is only enhancing the edges of original image .

\section{CONClusions}

This paper proposed a method of medical image enhancement based upon non-linear enhancement technique using Histogram equalization and the Discrete wavelet transform. These techniques enhance the lower $\&$ higher contrast area of an image in both spatial \& frequency domain. Here we compared these techniques based on MSE \& PSNR \& got better result of PSNR \& MSE in the case of preprocessed histogram technique as well as DWT results is also better than the simple histogram technique. Here Discrete wavelet Transform enhanced the edges of the image, So we can say that the DWT is mainly using for de-noising. Result of both experiments shows that the algorithm is not only enhancing the image contrast, but can preserving the original image quality.

\section{REFERENCES}

[1] Mr. Salem Saleh Al-amri1,Dr.N.V.Kalyankar2,Dr.S.D.Khamitkar3, Linear and Non linear Contrast Enhancement Image , IJCSNS International Journal of Computer Science and Network Security, VOL.10 No.2, February 2010.

[2] Sos S. Agaian, Blair Silver and L. Karen A. Panetta, ,Transform coefficient histogram-based image enhancement algorithms using contrast entropy, IEEE Trans. On Image Process, vol. 16,no. 3, Mar. 2007.

[3] Lu, J., Healy, D.M., and Weaver, J.B.: ,Contrast enhancement of medical images using multi-scale edge representation, Opt. Eng., 1994, 33, (7), pp. 2151-2161

[4] Yang, G., and Hansell, D.M.: ,CT image enhancement with wavelet analysis for the detection of small airways disease, IEEE Trans Med. Imaging, 1997, 16, (6), pp. 953-961

[5] Hasan Demirel and Gholamreza Anbarjafar, Discrete Wavelet Transform-Based Satellite Image Resolution Enhancement, IEEE Transactions On Geoscience And Remote Sensing, Vol. 49, No. 6, June 2011

[6] R. C. Gonzalez and R. E. Woods, Digital Image Processing (2nd Edi-tion). Prentice Hall, January 2002.

[7] Mallat, S.G.: , Multifrequency channel decompositions of image and wavelet models, IEEE Trans. Acoust. Speech Signal Process., 1989, 37, (12), pp. 2091-2110

[8] Akhil Pratap Singh, Agya Mishra,, Wavelet Based Watermarking On Digital Image, Akhil Pratap Singh Et. Al../ Indian Journal Of Computer Science And Engineering Vol 1 No 2, 86-91. 